(1)

CrossMark

\title{
Pulmonary rehabilitation in bronchiectasis: a propensity-matched study
}

\author{
Suhani Patel $\mathbb{1}^{1,2,6}$, Aaron D. Cole ${ }^{2,6}$, Claire M. Nolan ${ }^{1,2,3}$, Ruth E. Barker ${ }^{1,2}$, \\ Sarah E. Jones ${ }^{1,2}$, Samantha Kon ${ }^{1,3}$, Julius Cairn ${ }^{4}$, Michael Loebinger ${ }^{3,5}$, \\ Robert Wilson ${ }^{3,5}$ and William D-C. Man ${ }^{1,2,3,4}$
}

\begin{abstract}
Affiliations: ${ }^{1}$ Harefield Pulmonary Rehabilitation and Muscle Research Group, Royal Brompton and Harefield NHS Foundation Trust, Harefield, UK. ${ }^{2}$ Harefield Pulmonary Rehabilitation Unit, Royal Brompton and Harefield NHS Foundation Trust, Harefield, UK. ${ }^{3}$ National Heart and Lung Institute, Imperial College London, London, UK. ${ }^{4}$ Dept of Respiratory Medicine, Harefield Hospital, Royal Brompton and Harefield NHS Foundation Trust, Harefield, UK. ${ }^{5}$ Host Defence Unit, Royal Brompton and Harefield NHS Foundation Trust, London, UK. ${ }^{6}$ These two authors contributed equally to this work.
\end{abstract}

Correspondence: Suhani Patel, Harefield Pulmonary Rehabilitation and Muscle Research Group, Harefield Hospital, Harefield, UB9 6JH, UK. E-mail: s.patel1Arbht.nhs.uk

@ERSpublications

Patients with bronchiectasis benefit from pulmonary rehabilitation to the same extent as do patients with COPD, other than in the Fatigue domain of the CRQ. Patients with bronchiectasis should be referred for pulmonary rehabilitation. http://ow.ly/fQua30mOlPw

Cite this article as: Patel S, Cole AD, Nolan CM, et al. Pulmonary rehabilitation in bronchiectasis: a propensity-matched study. Eur Respir J 2019; 53: 1801264 [https://doi.org/10.1183/13993003.01264-2018].

ABSTRACT International guidelines recommend pulmonary rehabilitation for patients with bronchiectasis, supported by small trials and data extrapolated from chronic obstructive pulmonary disease (COPD). However, it is unknown whether real-life data on completion rates and response to pulmonary rehabilitation are similar between patients with bronchiectasis and COPD.

Using propensity score matching, 213 consecutive patients with bronchiectasis referred for a supervised pulmonary rehabilitation programme were matched 1:1 with a control group of 213 patients with COPD. Completion rates, change in incremental shuttle walk (ISW) distance and change in Chronic Respiratory Disease Questionnaire (CRQ) score with pulmonary rehabilitation were compared between groups.

Completion rate was the same in both groups (74\%). Improvements in ISW distance and most domains of the CRQ with pulmonary rehabilitation were similar between the bronchiectasis and COPD groups (ISW distance: 70 versus 63 m; CRQ-Dyspnoea: 4.8 versus 5.3; CRQ-Emotional Function: 3.5 versus 4.6; CRQ-Mastery: 2.3 versus 2.9; all $\mathrm{p}>0.20$ ). However, improvements in CRQ-Fatigue with pulmonary rehabilitation were greater in the COPD group (bronchiectasis 2.1 versus COPD 3.3; $\mathrm{p}=0.02$ ).

In a real-life, propensity-matched control study, patients with bronchiectasis show similar completion rates and improvements in exercise and health status outcomes as patients with COPD. This supports the routine clinical provision of pulmonary rehabilitation to patients with bronchiectasis. 


\section{Introduction}

Bronchiectasis is a chronic lung condition, characterised clinically by dyspnoea, productive cough and recurrent respiratory infections, and radiologically by abnormal and permanent dilatation of the bronchi [1]. Important management objectives include the reduction of symptoms (e.g. dyspnoea and exercise intolerance) and improvement in health-related quality of life (HRQoL).

Pulmonary rehabilitation, a multidisciplinary programme of care comprising individualised exercise training and education, is an integral component of the non-pharmacological management of patients with chronic respiratory disease, especially chronic obstructive pulmonary disease (COPD). Substantial data support the positive benefits of pulmonary rehabilitation on exercise capacity and HRQoL in patients with COPD, with the most recent Cochrane systematic review including 65 randomised controlled trials (RCTs) involving 3822 participants [2]. Although international guidelines recommend the provision of pulmonary rehabilitation in symptomatic patients with bronchiectasis, there is much less supporting evidence [1].

A recent systematic review of RCTs of pulmonary rehabilitation or exercise training in patients with bronchiectasis identified only four small trials [3]. Short-term improvements in exercise capacity and HRQoL were demonstrated with pulmonary rehabilitation or exercise training, but there was significant heterogeneity in the interventions delivered, and the two trials that contributed the majority of patients to the systematic review $[4,5]$ would not fulfil current consensus definitions of pulmonary rehabilitation $[6,7]$. Furthermore, there is limited real-life pulmonary rehabilitation data in patients with bronchiectasis, particularly with regard to adherence and completion rates, and it is not known whether they respond to pulmonary rehabilitation in a similar manner and magnitude as patients with COPD.

As the majority of evidence supporting the benefits of pulmonary rehabilitation comes from patients with COPD, the aim of our study was to compare the real-life responses of patients with bronchiectasis with a matched control group of patients with COPD to supervised outpatient pulmonary rehabilitation. Specifically, we aimed not only to compare the response to pulmonary rehabilitation in terms of changes in exercise capacity and HRQoL, but also to compare adherence and completion rates. We hypothesised that patients with bronchiectasis would have similar adherence and completion rates, as well as similar improvements in exercise capacity and HRQoL with pulmonary rehabilitation as matched patients with COPD.

\section{Methods}

Participants

Participants with bronchiectasis were prospectively recruited from community- and hospital-based pulmonary rehabilitation assessment clinics in northwest London (UK) between January 2012 and December 2016. Inclusion criteria were: a primary diagnosis of clinically significant bronchiectasis determined by a respiratory physician, with supporting evidence from thin-section computed tomography (CT) of bronchial dilatation (internal airway lumen greater than adjacent pulmonary artery; absence of tapering; airway visibility within $1 \mathrm{~cm}$ of pleural surface), stable disease (no change in medication for at least 6 weeks) and functional limitation due to breathlessness (defined as a Medical Research Council $(\mathrm{MRC})$ Dyspnoea score $\geqslant 2$ ). Exclusion criteria were: a coexisting diagnosis of COPD or a self-reported smoking history of $\geqslant 10$ pack-years to avoid possible contamination with coexisting COPD, or a coexisting history of cystic fibrosis.

The control group was recruited retrospectively from the pulmonary rehabilitation database and comprised patients with COPD [8] referred to the same pulmonary rehabilitation assessment clinics over the same time period, with a minimum 10 pack-year smoking history. They were propensity score matched 1:1 [9] accounting for age, sex, forced expiratory volume in $1 \mathrm{~s}$ (FEV1) \% pred, body mass index (BMI), MRC Dyspnoea score and baseline exercise capacity (measured by the incremental shuttle walk (ISW) test). Matching was performed by researchers blinded to completion or outcome data. Patients with a known coexisting diagnosis of bronchiectasis were excluded from the control group.

For both groups, those with a contraindication to exercise training (e.g. unstable ischaemic heart disease or severe aortic stenosis) were excluded. All patients provided informed consent to participate in pulmonary rehabilitation, and for their anonymised data to be used for service evaluation and audit purposes. Service evaluation and audit does not require local research ethical approval, as per guidance from the NHS Health Research Authority. All procedures performed in this study were part of standard clinical care.

\section{Pulmonary rehabilitation}

Both groups were offered an outpatient pulmonary rehabilitation programme consisting of two supervised sessions and at least one additional home session each week (total 16 supervised sessions) for 8 weeks. The 
programme was conducted according to British Thoracic Society quality standards for pulmonary rehabilitation [6]. Supervised sessions comprised $1 \mathrm{~h}$ of exercise and 45-60 $\mathrm{min}$ of education. Exercise training was individualised, reviewed and progressed at each supervised session, and comprised aerobic walking and cycling as well as upper and lower limb resistance training. Exercise training sessions were supervised by experienced respiratory physiotherapists with a minimum of 12 months dedicated specialist experience in pulmonary rehabilitation. A fuller description of the initial prescriptions for aerobic and resistance exercise for this pulmonary rehabilitation programme has been outlined previously [10]. Education sessions were delivered by a multidisciplinary team with topics chosen to develop patients' understanding and holistic management of their disease. This included importance of exercise and physical activity, medication use, inhaler technique, diet, coping and relaxation strategies, airway clearance, managing infections with early recognition, oxygen therapy, end-of-life care, and self-management plans for exacerbations.

\section{Measurements and outcomes}

Baseline assessments included BMI, ISW distance [11], spirometry (EasyOne spirometer; ndd Medical Technologies, Andover, MA, USA), MRC Dyspnoea score [12] and HRQoL (Chronic Respiratory Disease Questionnaire (CRQ) score) [13]. The ISW distance, MRC Dyspnoea score and CRQ score were also measured after completion of the 8-week pulmonary rehabilitation course. The ISW test was performed on a 10-m course according to international technical standards [14].

Pulmonary rehabilitation adherence was defined as the number of supervised sessions attended, while completion was defined as attendance at a minimum of eight supervised sessions [15] and the end of programme assessment.

\section{Analysis}

Data analyses were performed using Prism version 7 (GraphPad, La Jolla, CA, USA) and SPSS version 24 (IBM, Armonk, NY, USA). A p-value of $<0.05$ was considered statistically significant. Paired t-tests or Wilcoxon signed-rank tests were used to compare within-group response to pulmonary rehabilitation and two sample t-tests or Mann-Whitney tests were used to compare between-group responses to pulmonary rehabilitation. Chi-squared tests were used to compare completion and attendance rates.

\section{Results}

Between January 2012 and December 2016, 548 adults with a primary diagnosis of bronchiectasis were referred for pulmonary rehabilitation. Of these, 335 were excluded from the study because of the absence of a diagnostic CT scan $(n=41)$, a coexisting diagnosis of COPD ( $n=188)$, no coexisting diagnosis of COPD but smoking pack-year history $\geqslant 10(n=37)$, coexisting cardiac comorbidity that precluded exercise training $(n=14)$ and decline of the offer of pulmonary rehabilitation $(n=55)$ (summarised in figure 1). The resulting 213 patients with bronchiectasis were matched 1:1 with patients with COPD. Baseline characteristics are shown in table 1 . Bronchiectasis and COPD groups were well matched in terms of age, sex, BMI, MRC Dyspnoea score, FEV1 \% pred, baseline ISW distance and all domains of the CRQ. In patients with bronchiectasis, the $\mathrm{FEV} 1$ /forced vital capacity ratio, as expected, was higher than in patients with COPD (table 1).

In both groups, 157 patients completed the pulmonary rehabilitation programme (completion rate $74 \%$; $\mathrm{p}=1.0$ ) and the mean \pm SD number of sessions attended was similar (bronchiectasis $13 \pm 3$ versus COPD 13 \pm 2 ; $\mathrm{p}=0.54$ ). The most frequent cause for noncompletion in both groups was exacerbation of their chronic respiratory disease (bronchiectasis $\mathrm{n}=38$; $\operatorname{COPD} \mathrm{n}=35$ ) (figure 1 ).

Significant improvements in ISW and all domains of the CRQ with pulmonary rehabilitation were observed in both the bronchiectasis and COPD group (table 2 and figure 2). There were no between-group differences in ISW distance change or change in Dyspnoea, Emotional Function and Mastery domains in the CRQ. However, the improvement in CRQ-Fatigue was significantly less in the bronchiectasis group compared with the COPD group (table 2 and figure 2).

\section{Discussion}

In this observational, real-life propensity-matched control study, we have demonstrated that patients with bronchiectasis, uncontaminated by coexisting COPD, show significant improvements in exercise capacity and HRQoL to an 8-week outpatient supervised pulmonary rehabilitation programme. Furthermore, we have demonstrated that these improvements, as well as adherence and completion rates, are very similar to those observed in a propensity-matched control group of patients with COPD. These data add to the growing evidence to support the routine provision of pulmonary rehabilitation to patients with bronchiectasis and exertional dyspnoea. 


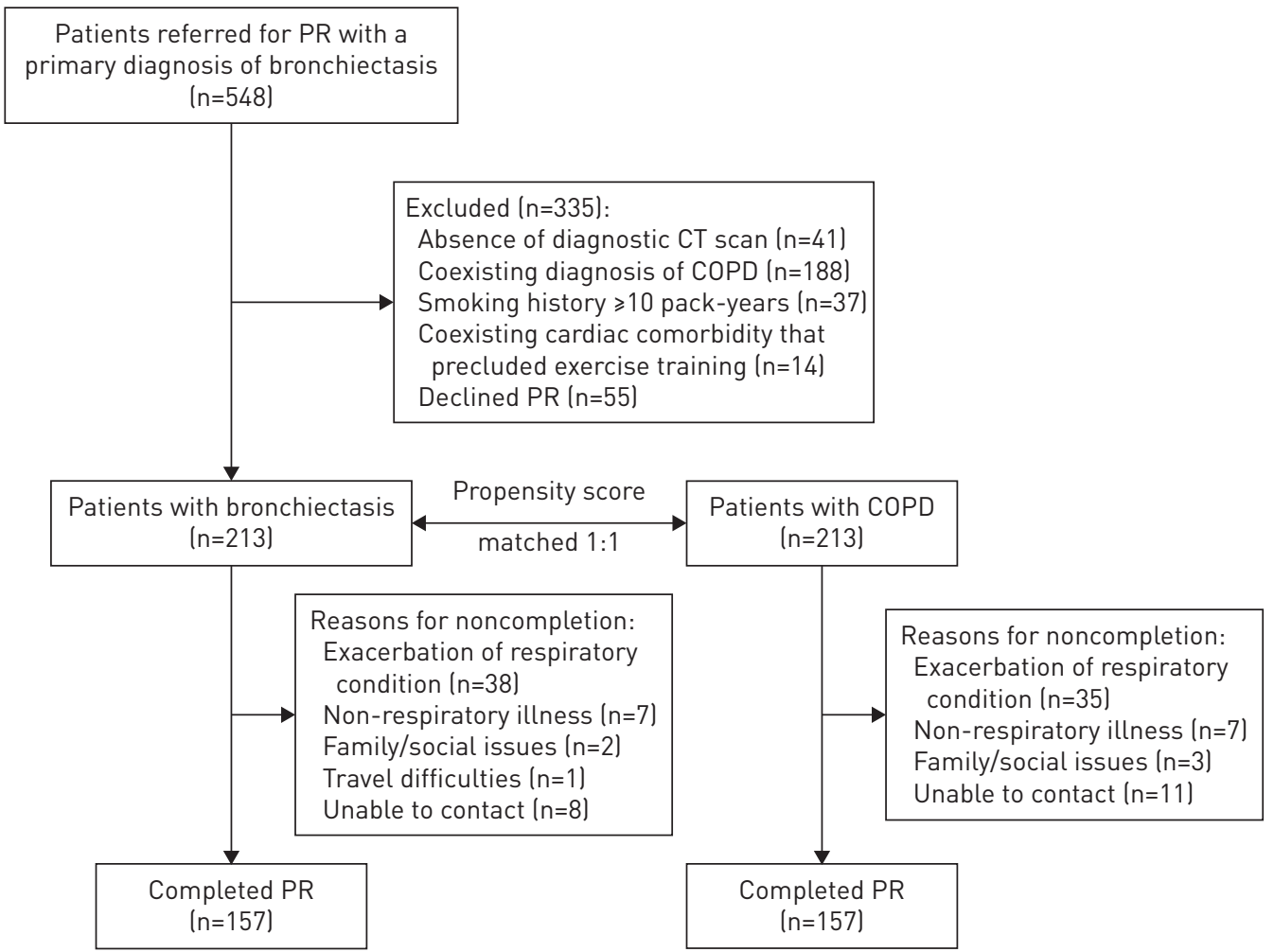

FIGURE 1 Participant recruitment and reasons for noncompletion of pulmonary rehabilitation (PR). CT: computed tomography; COPD: chronic obstructive pulmonary disease.

\section{Previous studies}

Whereas the evidence base supporting the provision of pulmonary rehabilitation in patients with COPD is substantial (65 RCTs involving 3822 patients [2]), it is considerably smaller in patients with bronchiectasis. In a recent systematic review of exercise-based interventions in bronchiectasis, LEE et al. [3] were only able to identify four RCTs, incorporating 164 patients (of who half underwent exercise training or pulmonary rehabilitation). Of these four trials, two comprised interventions that would not fulfil current consensus definitions of pulmonary rehabilitation [6,7]; the study by LeE et al. [4] included exercise training only with no educational component, whereas the intervention described by GREENING et al. [5] was a multimodal inpatient rehabilitation intervention of short duration during a severe exacerbation requiring hospitalisation followed by a minimally supervised home self-management plan that was considered "light-touch" [16]. There was variable study quality and sample sizes were small. For example, the largest trial [4] randomised 42 patients with bronchiectasis to exercise training with only 32 completing. Observational studies have included larger numbers of patients with bronchiectasis undergoing pulmonary rehabilitation. ZANINI et al. [17] described 108 patients with bronchiectasis undergoing a 3-week inpatient pulmonary rehabilitation program, and demonstrated improvements in 6-min walk distance (6MWD), generic HRQoL and dyspnoea scores. However, the study was retrospective and lacked a control group, which limited interpretation of findings. ONG et al. [18] studied a similar number of patients with bronchiectasis $(n=111)$ across two institutions, of whom $27 \%$ had coexisting pulmonary diseases, mainly COPD. 95 patients with bronchiectasis completed pulmonary rehabilitation across the two institutions, and combined results showed statistically and clinically important improvements in 6MWD and CRQ [18]. This study incorporated a control group of patients with COPD; although the authors showed similar improvements in 6MWD and CRQ-Total score in the bronchiectasis and COPD groups, they did not compare the individual CRQ domain scores (the conventional way of reporting this questionnaire) [13].

\section{Strengths and significant findings}

To the best of our knowledge, our study includes the largest described cohort of patients with bronchiectasis to undergo pulmonary rehabilitation and therefore adds to the existing literature. Other than sample size, other notable features of this study were inclusion criteria that included a diagnosis of bronchiectasis according to consensus guidelines [19], exclusion of coexisting COPD or those with significant smoking pack-year history from the bronchiectasis group and the inclusion of a carefully 


\begin{tabular}{lccc} 
TABLE 1 Baseline characteristics & & & \\
& Bronchiectasis & COPD & p-value \\
\hline Subjects & 213 & 213 & 0.48 \\
Age years & $70 \pm 11$ & $71 \pm 10$ & 0.84 \\
Male & $77(36)$ & $79(37)$ & $<0.0001$ \\
FEV1/FVC & $0.68 \pm 0.14$ & $0.55 \pm 0.12$ & 0.49 \\
FEV1 \% pred & $68.1 \pm 25.7$ & $66.5 \pm 22.1$ & 0.50 \\
MRC Dyspnoea score & $3 \pm 1$ & $3 \pm 1$ & 0.93 \\
Body mass index kg-m ${ }^{-2}$ & $27.1 \pm 6.11$ & $27.2 \pm 6.4$ & $<0.0001$ \\
Smoking history pack-years & $0(0-8)$ & $35(18-50)$ & 0.21 \\
Exacerbations in past year $\mathbf{n}$ & $2(0-4)$ & $1.5(1-3)$ & 0.54 \\
ISW distance m & $250(120-390)$ & $255 \pm 180$ & 0.27 \\
CRQ-Dyspnoea score & $15.6 \pm 5.8$ & $15.0 \pm 6.2$ & 0.61 \\
CRQ-Fatigue score & $13.9 \pm 4.9$ & $14.1 \pm 5.1$ & 0.68 \\
CRQ-Emotional Function score & $30.8 \pm 8.7$ & $31.2 \pm 9.3$ & 0.62 \\
CRQ-Mastery score & $19.1 \pm 5.5$ & $18.8 \pm 5.7$ & 0.84 \\
CRQ-Total score & $79.4 \pm 19.8$ & $79.0 \pm 21.6$ & \\
\hline
\end{tabular}

Data are presented as $\mathrm{n}, \mathrm{n}(\%)$, mean $\pm \mathrm{SD}$ or median (interquartile range), unless otherwise stated. COPD: chronic obstructive pulmonary disease; FEV1: forced expiratory volume in $1 \mathrm{~s}$; FVC: forced vital capacity; MRC: Medical Research Council; ISW: incremental shuttle walk; CRQ: Chronic Respiratory Disease Questionnaire.

matched control group of patients with COPD using a formal validated statistical technique (propensity score matching [9]) to minimise imbalance in key covariates between the groups. The intervention was a standardised pulmonary rehabilitation programme that adhered to national quality standards [6], run by experienced respiratory physiotherapists with a specialist interest in pulmonary rehabilitation. We demonstrated that patients with bronchiectasis had similar adherence and completion rates to pulmonary rehabilitation as matched patients with COPD, alleviating concerns of higher dropout from pulmonary rehabilitation in a chronic inflammatory condition characterised by recurrent exacerbations. Furthermore, we showed substantial (and similar) improvements in walking exercise capacity in both bronchiectasis and COPD groups. The mean improvements in ISW distance of 70 and $63 \mathrm{~m}$ in the bronchiectasis and COPD groups, respectively, are greater than the minimum clinically important difference (MCID) established in patients with chronic respiratory disease, such as COPD, bronchiectasis and idiopathic pulmonary fibrosis [20-22], and similar to that observed in an RCT of exercise training in bronchiectasis [4].

We also observed improvements in HRQoL with pulmonary rehabilitation in the bronchiectasis group. We used the CRQ, a well-validated and responsive questionnaire that is widely used in pulmonary rehabilitation and incorporates four domains (Dyspnoea, Fatigue, Emotional Function and Mastery). The established MCID for the CRQ is mean 0.5 points change per question of each domain [23]. All four CRQ domains showed statistically and clinically significant improvements, in contrast to a previous trial that was unable to demonstrate improvements in the Emotional Function and Mastery domains [4]. This is likely to be due to differences between the interventions; in particular, the intervention in the study of LeE et al. [4] included exercise training but no education programme. A previous observational study also demonstrated improvements in the CRQ in patients with bronchiectasis undergoing a comprehensive pulmonary rehabilitation, but did not compare CRQ domain scores with a matched control group of patients with

\begin{tabular}{lccc}
\hline \multicolumn{2}{l}{ TABLE 2 Response to pulmonary rehabilitation } & \\
& \multicolumn{1}{l}{ Bronchiectasis } & COPD & p-value \\
\hline Subjects & 157 & 157 & 0.36 \\
ISW distance m & $70(58-84)$ & $63(50-76)$ & 0.27 \\
CRQ-Dyspnoea score & $4.8(3.8-5.8)$ & $3.3(4.5-6.4)$ & 0.02 \\
CRQ-Fatigue score & $2.1(1.5-2.8)$ & $4.6(3.6-4.0)$ & 0.22 \\
CRQ-Emotional Function score & $3.5(2.3-4.7)$ & $2.9(2.1-3.6)$ & 0.26 \\
CRQ-Mastery score & $2.3(1.5-3.0)$ & $16.2(13.5-18.9)$ & 0.09 \\
CRQ-Total score & $12.8(9.9-15.6)$ & &
\end{tabular}

Data are presented as $\mathrm{n}$ or mean change $(95 \% \mathrm{CI})$, unless otherwise stated. COPD: chronic obstructive pulmonary disease; ISW: incremental shuttle walk; CRQ: Chronic Respiratory Disease Questionnaire. 

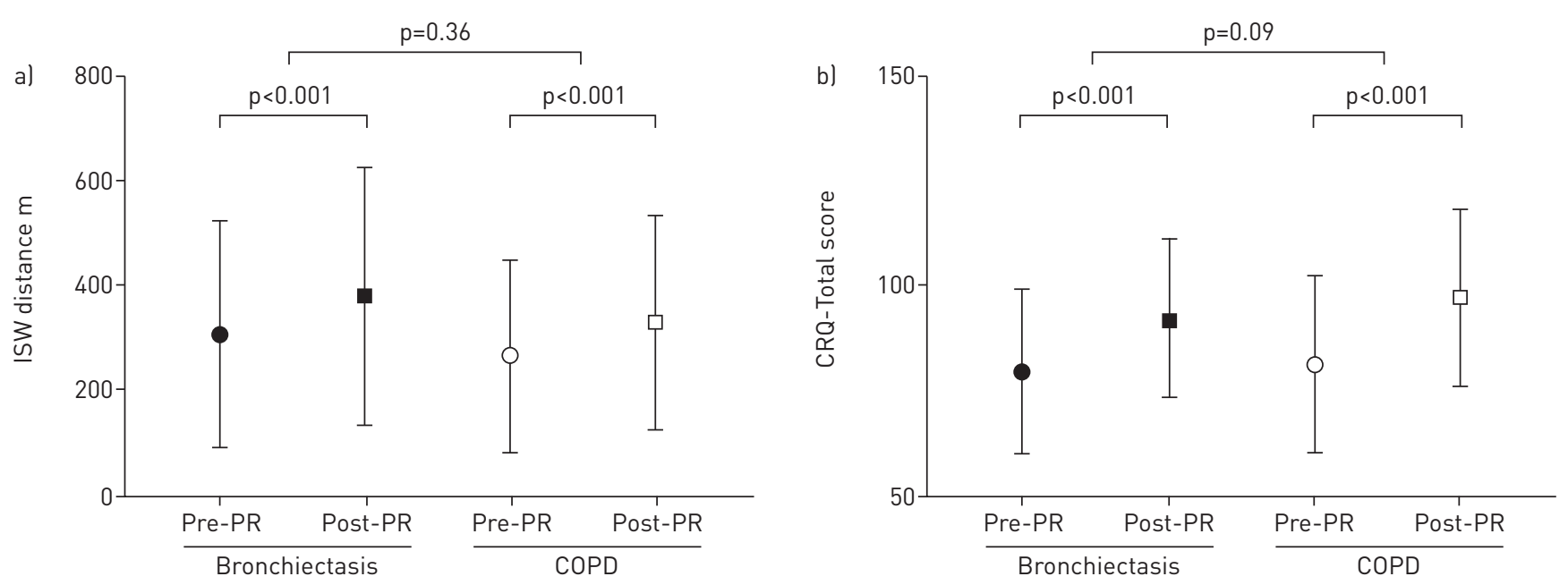

FIGURE 2 Response of a) incremental shuttle walk (ISW) distance and b) Chronic Respiratory Disease Questionnaire (CRQ)-Total score to pulmonary rehabilitation (PR). COPD: chronic obstructive pulmonary disease.

COPD. Our study showed that pulmonary rehabilitation produced similar improvements in three CRQ domains (Dyspnoea, Emotional Function and Mastery) and the CRQ-Total score in the bronchiectasis and COPD groups. For these measures, although there appeared to be numerically greater changes in the COPD group, the observed differences in change were considerably smaller than the established MCID [23]. However, the improvement in the CRQ-Fatigue domain with pulmonary rehabilitation was statistically less and blunted in the bronchiectasis group compared with the COPD group. This may reflect the differing psychometric properties of this questionnaire domain in patients with bronchiectasis and COPD. However, it may also reflect a characteristic symptom of bronchiectasis that may need additional attention than that provided by a conventional outpatient pulmonary rehabilitation programme. Previous studies have observed that $45-73 \%$ of patients with bronchiectasis have significant levels of fatigue [24], but relatively little consideration has been given to the pharmacological or non-pharmacological management of this symptom in bronchiectasis. Fatigue in bronchiectasis is complex; a correlation with anxiety and depression [25] has been observed, but not with markers of inflammation [26]. To the best of our knowledge, there are no published reports looking at the association between fatigue and disease severity on imaging. We propose fatigue in bronchiectasis is an area that warrants further study.

\section{Limitations}

Although our study was not a randomised study, our deliberate aim was to provide real-life data outside of carefully controlled research trials. We also used a validated statistical method to carefully match our cohort, and baseline characteristics, including anthropometry, exercise capacity, symptom scores and HRQoL, were very similar between the bronchiectasis and COPD groups (table 1). The study cohort also comprised an older bronchiectasis cohort, which may reduce the applicability of our findings across a wider age range. Although we tried to avoid contamination of the bronchiectasis group with coexisting COPD by excluding significant ex- or current smokers, for the COPD control group we relied on clinician diagnosis and did not have CT evidence to exclude the possibility that some of the COPD group had coexisting bronchiectasis. In our study, we had contemporaneous sputum microbiology data in only a small number of the cohort. We were aware of only 12 patients with sputum Pseudomonas aeruginosa colonisation. Our study focused on the immediate improvements in exercise capacity and HRQoL with pulmonary rehabilitation, as is routinely recorded in clinical practice, but did not report on long-term follow-up. We are therefore unable to comment on the duration of the effects of pulmonary rehabilitation in bronchiectasis (and how this compares with COPD).

\section{Future work}

Although the provision of pulmonary rehabilitation is recommended in international bronchiectasis guidelines [1, 19], there remains a paucity of RCTs, particularly in patients with acute exacerbations requiring hospitalisation. Little data exist on the cost-effectiveness and health economic implications of providing pulmonary rehabilitation to patients with bronchiectasis, and longer-term data are needed to evaluate whether pulmonary rehabilitation has an impact on outcomes and end-points such as exacerbations and mortality. Our work identified that fatigue symptoms do not seem to be as responsive to pulmonary rehabilitation in patients with bronchiectasis compared with COPD and this warrants further investigation. 


\section{Conclusions}

This real-life cohort study confirms the findings from previous clinical trials demonstrating significant improvements in exercise capacity and HRQoL in patients with bronchiectasis undergoing supervised pulmonary rehabilitation. These improvements, as well as adherence and completion rates, are of similar magnitude to those observed in a matched group of patients with COPD. The data adds to the growing evidence base to support the routine clinical provision of pulmonary rehabilitation to patients with bronchiectasis.

Conflict of interest: S. Patel has nothing to disclose. A.D. Cole has nothing to disclose. C.M. Nolan has nothing to disclose. R.E. Barker has nothing to disclose. S.E. Jones has nothing to disclose. S. Kon has nothing to disclose. J. Cairn has nothing to disclose. M. Loebinger has nothing to disclose. R. Wilson has nothing to disclose. W.D-C. Man reports grants from Pfizer, non-financial support from GSK, and personal fees from Mundipharma and Novartis, outside the submitted work.

\section{References}

1 Polverino E, Goeminne PC, McDonnell MJ, et al. European Respiratory Society guidelines for the management of adult bronchiectasis. Eur Respir J 2017; 50: 1700629.

2 McCarthy B, Casey D, Devane D, et al. Pulmonary rehabilitation for chronic obstructive pulmonary disease. Cochrane Database Syst Rev 2015; 2: CD003793.

3 Lee AL, Hill CJ, McDonald CF, et al. Pulmonary rehabilitation in individuals with non-cystic fibrosis bronchiectasis: a systematic review. Arch Phys Med Rehabil 2017; 98: 774-782.

4 Lee AL, Hill CJ, Cecins N, et al. The short and long term effects of exercise training in non-cystic fibrosis bronchiectasis - a randomised controlled trial. Respir Res 2014; 15: 44.

5 Greening NJ, Williams JE, Hussain SF, et al. An early rehabilitation intervention to enhance recovery during hospital admission for an exacerbation of chronic respiratory disease: randomised controlled trial. BMJ 2014; 349: g4315.

6 Bolton CE, Bevan-Smith EF, Blakey JD, et al. British Thoracic Society guideline on pulmonary rehabilitation in adults. Thorax 2013; 68: Suppl. 2, ii1-ii30.

7 Spruit MA, Singh SJ, Garvey C, et al. An official American Thoracic Society/European Respiratory Society statement: key concepts and advances in pulmonary rehabilitation. Am J Respir Crit Care Med 2013; 188: e13-e64.

8 Vogelmeier CF, Criner GJ, Martinez FJ, et al. Global strategy for the diagnosis, management and prevention of chronic obstructive lung disease 2017 report. Respirology 2017; 22: 575-601.

9 Rosenbaum PR, Rubin DB. The central role of the propensity score in observational studies for causal effects. Biometrika 1983; 70: 41-55.

10 Maddocks M, Kon SS, Canavan JL, et al. Physical frailty and pulmonary rehabilitation in COPD: a prospective cohort study. Thorax 2016; 71: 988-995.

11 Singh SJ, Morgan MD, Scott S, et al. Development of a shuttle walking test of disability in patients with chronic airways obstruction. Thorax 1992; 47: 1019-1024.

12 Fletcher CM, Elmes PC, Fairbairn AS, et al. Significance of respiratory symptoms and the diagnosis of chronic bronchitis in a working population. Br Med J 1959; 2: 257-266.

13 Guyatt GH, Berman LB, Townsend M, et al. A measure of quality of life for clinical trials in chronic lung disease. Thorax 1987; 42: 773-778.

14 Holland AE, Spruit MA, Troosters T, et al. An official European Respiratory Society/American Thoracic Society technical standard: field walking tests in chronic respiratory disease. Eur Respir J 2014; 44: 1428-1446.

15 Sewell L, Singh SJ, Williams JE, et al. How long should outpatient pulmonary rehabilitation be? A randomised controlled trial of 4 weeks versus 7 weeks. Thorax 2006; 61: 767-771.

16 Man WD, Kon SS, Maddocks M. Rehabilitation after an exacerbation of chronic respiratory disease. BMJ 2014; 349: g4370.

17 Zanini A, Aiello M, Adamo D, et al. Effects of pulmonary rehabilitation in patients with non-cystic fibrosis bronchiectasis: a retrospective analysis of clinical and functional predictors of efficacy. Respiration 2015; 89: 525-533.

18 Ong H, Lee A, Hill C, et al. Effects of pulmonary rehabilitation in bronchiectasis: a retrospective study. Chron Respir Dis 2011; 8: 21-30.

19 British Thoracic Society. BTS Quality Standards for Clinically Significant Bronchiectasis in Adults. 2012. www.brit-thoracic.org.uk/document-library/clinical-information/bronchiectasis/bts-quality-standards-for-clinicallysignificant-bronchiectasis-in-adults Date last accessed: December 4, 2018.

20 Singh SJ, Jones PW, Evans R, et al. Minimum clinically important improvement for the incremental shuttle walking test. Thorax 2008; 63: 775-777.

21 Lee AL, Hill CJ, Cecins N, et al. Minimal important difference in field walking tests in non-cystic fibrosis bronchiectasis following exercise training. Respir Med 2014; 108: 1303-1309.

22 Nolan CM, Delogu V, Maddocks M, et al. Validity, responsiveness and minimum clinically important difference of the incremental shuttle walk in idiopathic pulmonary fibrosis: a prospective study. Thorax 2018; 73: 680-682.

23 Jaeschke R, Singer J, Guyatt GH. Measurement of health status: ascertaining the minimal clinically important difference. Control Clin Trials 1989; 10: 407-415.

24 Hester K, Macfarlane J, Tedd H, et al. Fatigue in bronchiectasis. QJM 2011; 105: 235-240.

25 O'Leary C, Wilson C, Hansell D, et al. Relationship between psychological well-being and lung health status in patients with bronchiectasis. Respir Med 2002; 96: 686-692.

26 Wilson C, Jones P, O'Leary C, et al. Systemic markers of inflammation in stable bronchiectasis. Eur Respir J 1998; 12: $820-824$. 